\title{
Viability of Point Spread Function Deconvolution for SEM
}

\author{
Mandy C. Nevins ${ }^{1}$, Richard K. Hailstone ${ }^{1}$, Matthew D. Zotta ${ }^{2}$ and Eric Lifshin ${ }^{3}$ \\ 1. Center for Imaging Science, Rochester Institute of Technology, Rochester, NY. \\ 2. Nanojehm, Albany, NY. \\ ${ }^{3 .}$ SUNY Polytechnic Institute, Albany, NY.
}

An image is understood as the point spread function (PSF) of an imaging system convolved with the scene being imaged. An image can be restored to more accurately depict the scene by performing a deconvolution of the PSF and the image. In a scanning electron microscope (SEM), the PSF describes the shape of the electron beam. PSF deconvolution is a promising image restoration technique for SEM images [1] and is of special interest at low beam voltages $(<3 \mathrm{kV})$, where resolution is hindered by multiple factors. This technique's viability for SEM application is based on invariance of beam shape with respect to position and sample. Using Aura Workstation [2], we can determine the beam shape to test for invariance and perform restorations for image quality evaluation.

In this study, we investigate beam shape variation at different beam voltages 1) across multiple regions of the same sample and 2) between different samples. For these experiments, we capture backscattered electron images with a TESCAN MIRA3 field emission SEM (FESEM). In addition, we quantify the restoration provided by PSF deconvolution for comparison to image enhancement capabilities in commonly used software such as ImageJ, Adobe Photoshop, and RawTherapee. Our image quality metrics include ISO/TS 24597 on image sharpness in the SEM.

To study beam shape invariance, we image $19 \mathrm{~nm}$ gold nanoparticles on a carbon film transmission electron microscope (TEM) grid and on a substrate such as Kapton. To test for spatial invariance, we image at least six non-overlapping, same-sized regions the area of within one grid square. We start with a region of best focus and then keep the same beam shape for the following regions. To test for sample invariance, we image one region of best focus on either the TEM grid or the substrate and then, using the same beam shape, image a same-sized region on the other sample. Analyzing this data involves obtaining a PSF of each region using Aura and fitting a 2D Gaussian to each using MATLAB's curve fitting tool. The standard deviations of a beam, $\sigma_{\mathrm{x}}$ and $\sigma_{\mathrm{y}}$, can be used for comparison to other beams.

Beam shape variation at different voltages reflects our general understanding, being that beam diameter increases as beam voltage decreases (Figure 1). Noise becomes more prominent at low voltage as well, as seen by comparing the smooth surfaces of the higher $\mathrm{kV}$ beams to the rough surface of the $2 \mathrm{kV}$ beam. In the case of the TEM grid, our preliminary findings suggest the PSF is approximately spatially invariant regardless of beam voltage (Figure 2). Absolute percent differences between the beam shape standard deviations are shown in Table 1 for voltages depicted in Figure 2. We are currently undergoing study of spatial invariance for the substrate and beam shape invariance between the carbon TEM grid and the substrate. We are examining the image quality of Aura's restorations in comparison to enhancements done with ImageJ, Photoshop, and RawTherapee and other reference data imaged with the FESEM (Figure 3) [2]. 
References:

[1] Lifshin, E., et al, Microsc. Microanal. 20 (2014), p. 386.

[2] The authors acknowledge Nanojehm for the Aura Workstation beta unit on loan.

$20 \mathrm{kV}$

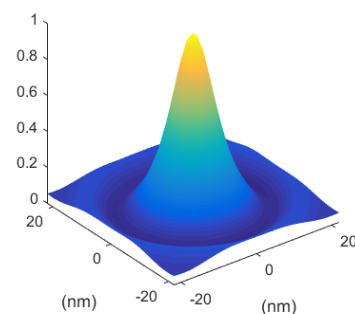

$10 \mathrm{kV}$

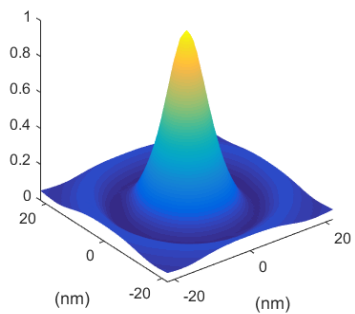

$5 \mathbf{k V}$

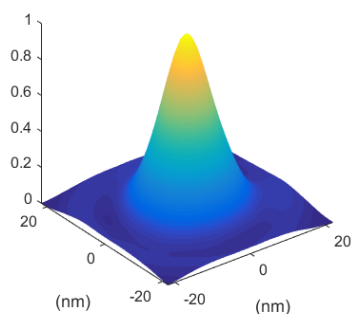

$2 \mathbf{k V}$

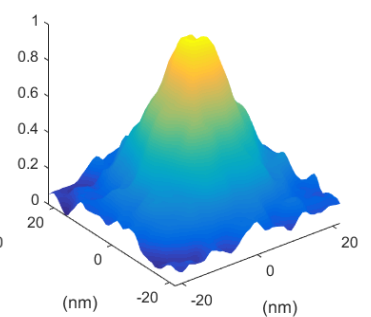

Figure 1. Beam Shape over a Range of Beam Voltages. These beam shapes were determined using the TEM grid sample. In general, as the beam energy decreases, the beam shape diameter increases. The PSFs show lowest to highest elevations as dark blue to light yellow, respectively.
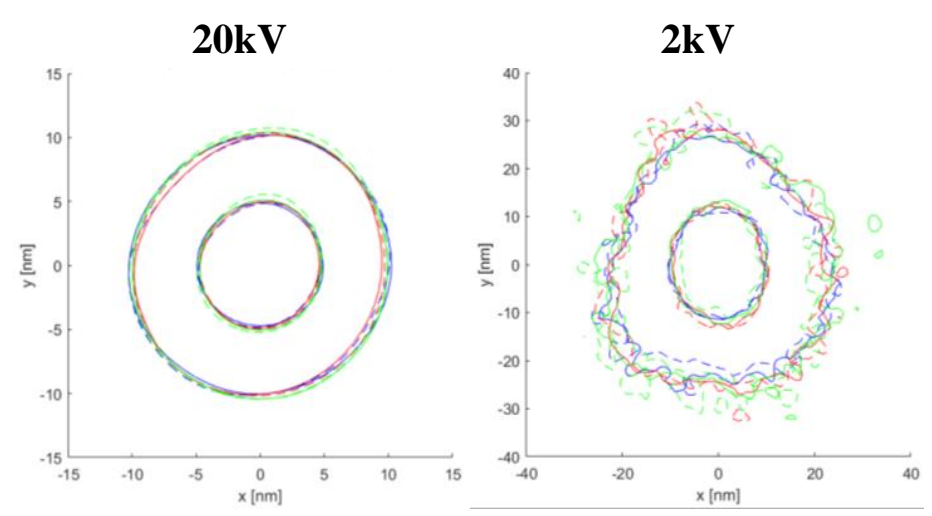

Figure 2. Comparison of Beam Shapes between Different Regions of the Same Sample. For each beam voltage, six non-overlapping regions in a TEM grid square are imaged. A contour plot representing the beam shape for each region is shown for $20 \mathrm{kV}$ on the left and $2 \mathrm{kV}$ on the right. The inner contour level represents the approximate FWHM (containing 40\% of probe current) and the outer contour level presents the approximate FWTM (containing $\sim 75 \%$ of probe current). Scale is different between $20 \mathrm{kV}$ and $2 \mathrm{kV}$.

\begin{tabular}{|c|c|c|c|c|}
\hline $\begin{array}{c}\text { Beam } \\
\text { Voltage }\end{array}$ & $\begin{array}{c}\boldsymbol{\sigma}_{\mathbf{x}} \\
\text { median }\end{array}$ & $\begin{array}{c}\boldsymbol{\sigma}_{\mathbf{x}} \\
\text { range }\end{array}$ & $\begin{array}{c}\boldsymbol{\sigma}_{\mathbf{y}} \\
\text { median }\end{array}$ & $\begin{array}{c}\boldsymbol{\sigma}_{\mathbf{y}} \\
\text { range }\end{array}$ \\
\hline $\mathbf{2 0 k V}$ & $2.0 \%$ & $0.6-5.5 \%$ & $3.6 \%$ & $0.5-8.5 \%$ \\
\hline $\mathbf{2 k V}$ & $5.3 \%$ & $0.0-13.6 \%$ & $3.8 \%$ & $0.4-9.9 \%$ \\
\hline
\end{tabular}

Table 1. Absolute Percent Difference between Beam Shapes for Different Regions of the Same Sample.

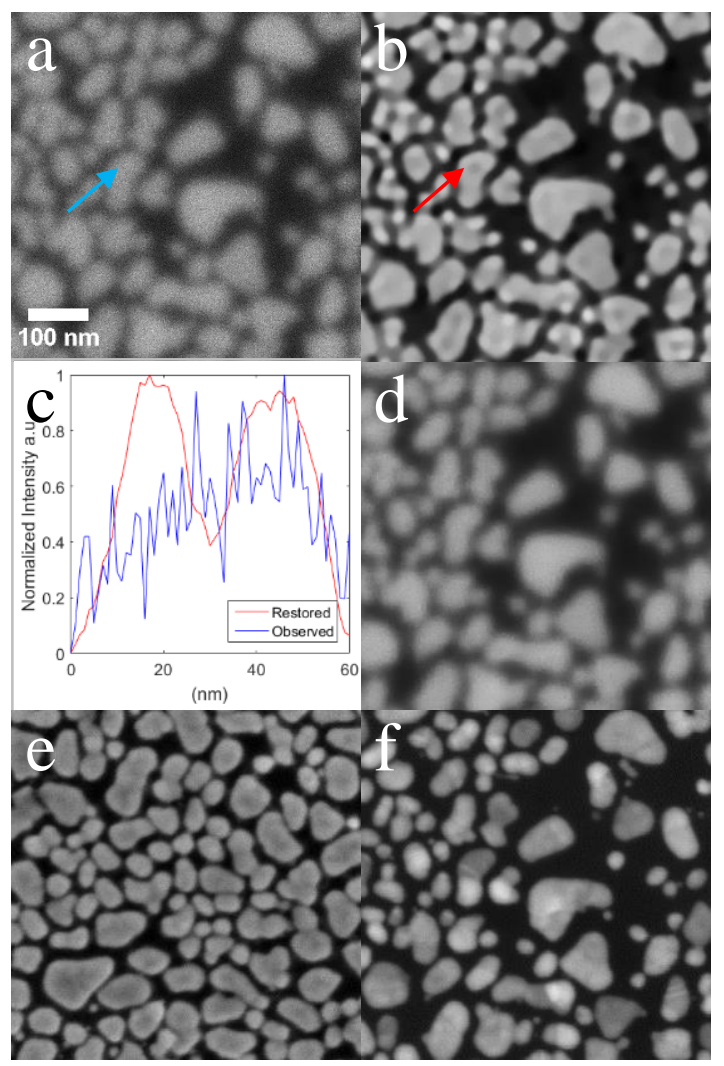

Figure 3. Visual Comparison of Low Voltage Gold on Carbon. a) observed at $2 \mathrm{kV}$, b) restoration of (a) with Aura, c) intensity profile of arrow in (a) and (b) where the blue line is the observed intensity and the red line is the restored intensity, d) enhancement of (a) with RawTherapee, e) similar field as (a) using beam deceleration at $2 \mathrm{kV}$, f) same field as (a) at $20 \mathrm{kV}$. Scale bar in (a) applies to all images. 